
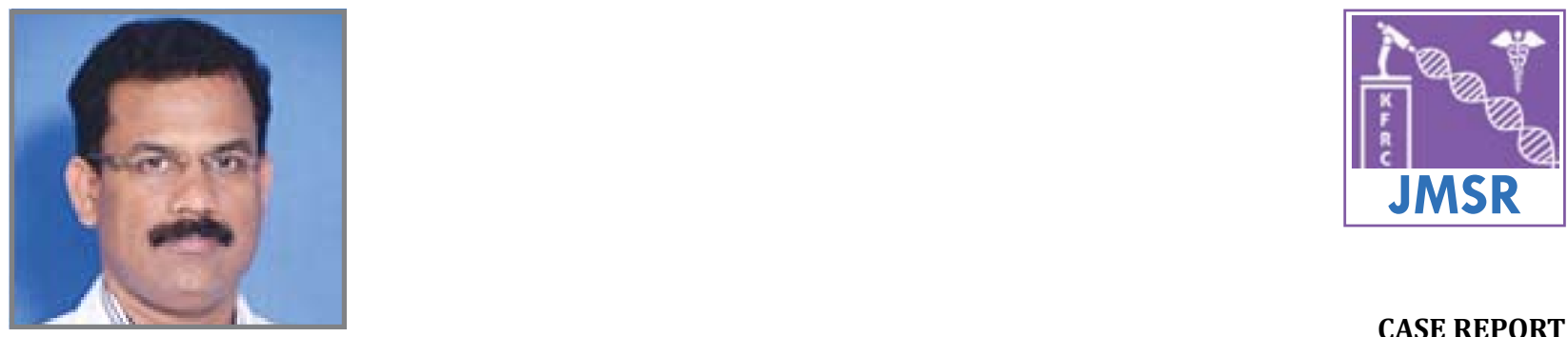

Dr. V.V. Ramana Prasad

CASE REPORT

\title{
Cryptogenic organising pneumonia an unusual presentation as solitary pulmonary nodule
}

Dr. V.V. Ramana Prasad ${ }^{1, *}$ and Dr. P. Kiran Kumar Reddy ${ }^{1}$

${ }^{1}$ Department of Pulmonology, Krishna Institute of Medical Sciences, Minister Road, Secunderabad-500003, Telangana, India

\begin{abstract}
Organizing pneumonia (OP) is well recognized, but the contexts in which it is encountered continue to increase. In parallel with an appreciation of new causes and associations of OP, increasing array of High-resolution computed tomography (HRCT) manifestations, an understanding of OP in the spectrum of lung injury and repair has evolved. Cryptogenic organising pneumonia (COP) presenting as solitary pulmonary nodule (SPN) is very unusual and this case of cryptogenic organising pneumonia is presented as solitary pulmonary nodule.
\end{abstract}

Keywords: Cryptogenic organising pneumonia; Bronchiolitis obliterance organising pneumonia; Solitary pulmonary nodule; Organizing pneumonia

*Corresponding author: Dr. Ramana Prasad VV, Consultant pulmonologist, Department of Pulmonology, Krishna Institute of Medical Sciences, Minister Road, Secunderabad-500003, Telangana, India.

Received 04 July 2014; Revised 03 September 2014; Accepted 13 September 2014

Citation: Ramana Prasad VV, Kiran Kumar Reddy P (2014) Cryptogenic organising pneumonia an unusual presentation as solitary pulmonary nodule. J Med Sci Res 2(4):200-202. DOI: http://dx.doi.org/10.17727/JMSR.2014/2-035

Copyright: (c) 2014 Ramana PrasadVV, et al. This is an open-access article distributed under the terms of the Creative Commons Attribution License, which permits unrestricted use, distribution, and reproduction in any medium, provided the original author and source are credited.

\section{Introduction}

Bronchiolitis obliterans organising pneumonia (BOOP) is a nonspecific response to various forms of lung injury and is the pathological hallmark of the distinct clinical entity [1]. It has been classified as cryptogenic (idiopathic) or secondary. BOOP may occur in association with connective tissue disease, with variable immunodeficiency syndrome, HIV infection, hepatitis viral infection, bacterial infections, graft vs. host disease and bone marrow transplant, with lung transplant, drug toxicity, radiation toxicity or it may be associated with lung tumours, ulcerative colitis and myelodysplastic syndrome.

\section{Case summary}

A 54-years-old female presented with complaints of dry cough since 40 days, no postural and diurnal variation, no aggravating and relieving factors, history of shortness of breath since one week, on minimal exertion functional class-III, history of fever low grade intermittent since 7 days. There was no 
history of chest pain, sweating, syncopal attacks, joint pains, no history of any drug exposure, no history of loss of appetite, loss of weight, hemoptysis. The patient denied toxic gas inhalations, contact with animals and patient did not have any connectivetissue disorder.

When patient came to the pulmonology department, KIMS Hospital, Secunderabad, her body temperature was $36.8^{\circ} \mathrm{C}$; respiratory rate was $21 / \mathrm{min}$; blood pressure was $140 / 80 \mathrm{~mm} / \mathrm{Hg}$ and heart rate was 86 beats/min. On examination, no lymphadenopathy was found and no abnormality detected on auscultation. In the radiograph of chest PA view showed solitary pulmonary nodule on left upper zone (Figure 1).

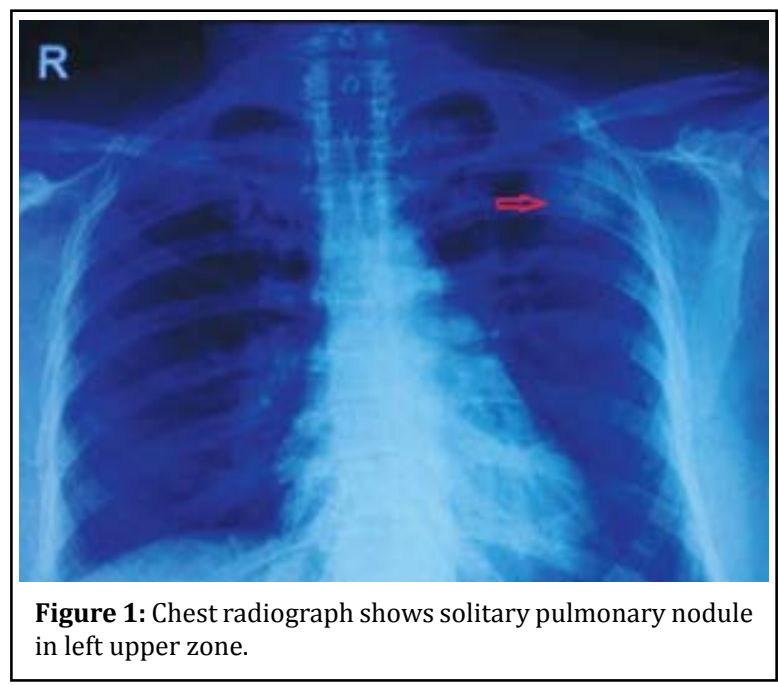

Computed tomography guided biopsy (Figure 2) was done from the lesion under local anaesthesia with all aseptic precautions. Procedure was done with 18G, $15 \mathrm{~cm}$ biopsy gun. Histopathology revealed vascularised granulation tissue occupying alveolar spaces, patchy interstitial collection of lymphocytes seen, features suggesting as bronchiolitis obliterans organising pneumonia. No infectious cause was found, connective tissue profile was normal, and so patient was treated with prednisolone $1 \mathrm{mg} / \mathrm{kg}$ body weight for one month along with other supportive measures. After one month chest radiograph PA view revealed resolution of left upper zone nodular lesion (Figure 3) now patient is on maintenance steroids.

\section{Discussion}

Epler et al., in 1985, provided the first description of BOOP as a clinicopathologic entity [2]. BOOP

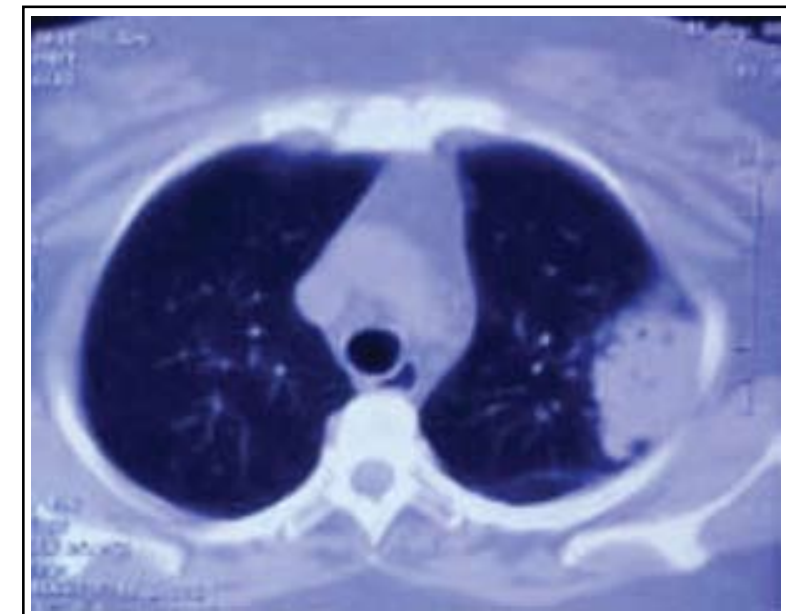

Figure 2: Computed tomography of the patient shows right lung lesion.

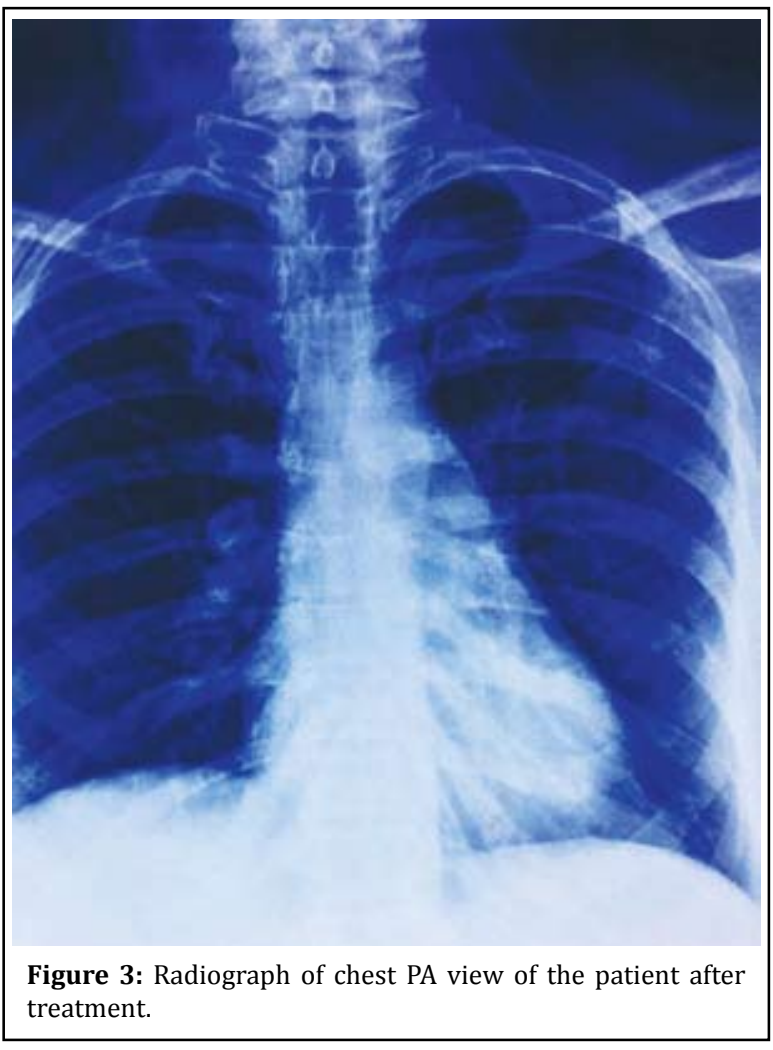

may be classified into three categories according to its etiology: organizing pneumonia of determined cause; organizing pneumonia of undetermined cause but occurring in a specific and relevant context, and cryptogenic (idiopathic) organizing pneumonia.

Bronchiolitis obliterans with organizing pneumonia (BOOP) is a rare lung condition in which the small airways (bronchioles) and air exchange sac (alveoli) become inflamed with connective tissue [3]. This is an uncommon illness occurring in 6 out of 100 , 
000 hospitalizations. It usually starts with a flu-like illness associated with fever, malaise, fatigue and cough. The cough may be persistent and troubling. There is shortness of breath with exertion and weight loss occurs in about half of patients.

This condition is a form of interstitial (within the fine supporting tissue of the lung) pneumonia of unknown origin. Very similar interstitial pneumonias can be seen in association with connective tissue diseases such as lupus erythematosus, several drug exposures and malignancies.

The physician examination is typical of other interstitial pneumonias but the chest radiograph and chest CT scan are distinctive and should lead an experienced lung specialist to suspect the diagnosis. However these findings are not definitive and a lung biopsy is recommended for confirmation. Pulmonary function tests are nonspecific. The course of the disease is variable however it tends to be persistent and not self-limited.

Current therapy involves relatively high doses of corticosteroids (e.g. Prednisone) for several months depending upon the response. Other immunosuppressive drugs (e.g. Cyclophosphamide) may also be used. Treatment is usually (but not always) results in significant improvement. However recurrences are common and patients should be periodically monitored with chest radiography, especially in the first year after treatment.

\section{Conclusion}

Solitary pulmonary nodule is a mass in lung smaller than 3 centimetres in diameter. The nodule most commonly represents a benign tumour, but in $20 \%$ cases represents malignant cancer. Cryptogenic organising pneumonia or bronchiolitis obliterans organising pneumonia is a pulmonary disorder with a wide spectrum of radiological features, usually these are bilateral, patchy, alveolar or ground glass infiltrates, but presenting as solitary pulmonary nodule is very unusual and there are very few cases reported in the literature.

\section{Acknowledgements}

Acknowledgements are due to Department of RadiologyandImaging,KIMSHospital,Secunderabad, Telangana, India.

\section{Conflict of interest}

The authors declare no conflict of interest.

\section{References}

1. White KA, Ruth-Sahd LA. Bronchiolitis obliterans organizing pneumonia. Crit Care Nurse 2007; 27(3):53-66.

2. Tasci C, Ozkan M, Karadurmus N, Naharci I, Cakar M, et al. Bronchiolitis obliterans with organizing pneumonia associated with Aspergillus fumigatus. Respir Med CME. 2011; 4(2):64-66.

3. Cryptogenic Organizing Pneumonia (COP) - American Lung Association. Available from: http://www.lung.org/lungdisease/cryptogenic-organizing-pneumonia 